\title{
Clinical Improvement Is Achieved Following Tibial Tubercle Distomedialization for Patellar Maltracking and Patella Alta Without Instability
}

Tarik Bayoumi, M.D., Dennis C. van Duijvenbode, M.D., Joyce L. Benner, Ph.D., Kirsten D. S. Boerma-Argelo, Michel H. J. Stavenuiter, M.D., and Jelle P. van der List, M.D.

\begin{abstract}
Purpose: To determine short-term patient-reported outcomes following distomedial tibial tubercle transfer (TTT) in patients with patellar maltracking and patella alta without instability. Methods: A single-surgeon case series study was performed on patients receiving distomedial TTT for the indication of patellar maltracking and patella alta without instability, after nonresponse to conservative treatment. Patient-reported outcomes were assessed preoperatively and at 3-, 6-, 12-, and 24-month follow-up using Kujala, Knee injury and Osteoarthritis Outcome Score (KOOS), and visual analog scale (VAS) pain scores. Generalized estimating equations analyses were performed to study improvement over time. Minimal clinically important differences obtained from literature were used to determine clinical relevance. Results: A total of 40 patients were included. Eight patients were lost to follow-up; thus, outcomes of 32 patients were analyzed. Mean follow-up was 22 months, median age was 21 years, and $75 \%$ were female. Mean Kujala score increased pre- to postoperatively from $55 \pm 12$ to $79 \pm 16(P<.001)$, KOOS from $48 \pm 14$ to $79 \pm 15(P<.001)$, and VAS from $64 \pm$ 17 to $25 \pm 21(P<.001)$, respectively. Eighty-four percent had clinical improvement of Kujala score, $91 \%$ of KOOS, and $78 \%$ of VAS score. A plateau phase in pain reduction was reached at 3 months, and in functional improvement at 6 months follow-up, after which no further significant improvement was observed. Complication rate was $3 \%$ and removal of hardware rate was $72 \%$. Conclusions: In this case series study, distomedial TTT led to clinically relevant functional improvement and pain reduction in patients with patellar maltracking and patella alta without instability. However, the removal of hardware rate was high $(72 \%)$. Level of Evidence: Level IV, therapeutic case series.
\end{abstract}

A nterior knee pain with related patellar maltracking is a common knee complaint, especially at younger age. ${ }^{1,2}$ The relation between anterior knee pain and patellar maltracking is much debated and is likely to be multifactorial. ${ }^{3-5}$ Patella alta refers to a

From the Department of Orthopedic Surgery, Northwest Clinics, Alkmaar, (T.B., J.L.B., K.D.S.A., M.H.J.S., J.P.v.d.L.); Centre for Orthopedic Research Alkmaar (CORAL), Alkmaar (T.B., D.C.v.D., J.L.B., K.D.S.A., M.H.J.S.); and Department of Orthopedic Surgery, Spaarne Gasthuis Hospital, Hoofddorp (J.P.v.d.L.), The Netherlands.

The authors report that they have no conflicts of interest in the authorship and publication of this article. Full ICMJE author disclosure forms are available for this article online, as supplementary material.

Tarik Bayoumi, M.D., and Dennis C. van Duijvenbode, M.D., share first authorship.

Received July 9, 2020; accepted January 27, 2021.

Address correspondence to Tarik Bayoumi, M.D., CORAL, P.O. Box 501, 1800 AM Alkmaar, the Netherlands.E-mail:tbayoumi.md@gmail.com

(C) 2021 THE AUTHORS. Published by Elsevier Inc. on behalf of the Arthroscopy Association of North America. This is an open access article under the CC BY-NC-ND license (http://creativecommons.org/licenses/by-nc-nd/4.0/). 2666-061X/201195

https://doi.org/10.1016/j.asmr.2021.01.027 high-riding patella and is often associated with patellar maltracking and subsequent anterior knee pain. ${ }^{3,4,6,7}$ The greater position of the patella in relation to the femoral trochlea is theorized to result in reduced osseous stability and lateral patellar tracking, as engagement with the trochlear groove occurs only at a greater knee flexion angle. ${ }^{2,6}$ Subsequently, patellar maltracking may lead to reduced patellar contact area during flexion, resulting in increased patellofemoral peak stress, pain during knee motion, and patellofemoral cartilage damage. ${ }^{8,9}$ In addition, patellar maltracking is believed to predispose to patellar subluxation and complete patellar dislocation. ${ }^{10}$

After nonresponse to conservative treatment, surgical management can be considered for patients with patellar maltracking with patella alta. ${ }^{5}$ Tibial tubercle transfer (TTT) is a distal realignment procedure performed to improve patellar tracking and can be individualized based on the patient-specific anatomic variances. $^{11,12}$ Hence, many variations of TTT have been described in the literature. ${ }^{5}$ For patients with patella alta, a distomedialization of the tibial tubercle aims 
to correct patellar tracking by transferring the tibial tubercle in distal and medial direction. ${ }^{13}$ Distomedial transfer distance in this procedure is based on correction of patellar height and tibial tubercle-trochlear groove (TT-TG) distance. ${ }^{5,14}$ TTT has been shown to be effective in the management of patella alta with recurrent dislocation but has not been studied extensively as treatment for patellar maltracking and patella alta without instability. ${ }^{15} \mathrm{~A}$ recent systematic review assessed all studies performing TTT for management of patellar maltracking without instability and found promising results in these patients. ${ }^{16}$ However, only a few studies have focused on TTT for the indication of patella alta, 4,17 and only one of them prospectively assessed outcomes in this population following TTT. ${ }^{4}$ The purpose of this study was to determine shortterm patient-reported outcomes following distomedial TTT in patients with patellar maltracking and patella alta without instability.

\section{Methods}

\section{Study Design}

From September 2012 until September 2015, a single-surgeon, case series study was performed in a large teaching hospital in Alkmaar, The Netherlands. Patients undergoing TTT for patellar maltracking with patella alta were screened for eligibility. Patients were eligible for inclusion if they had (1) patellar maltracking with presence of patella alta (i.e., Caton-Deschamps Index $[C D I] \geq 1.3$ ), ${ }^{18}$ (2) insufficient response to previous conservative treatment for at least 6 months (e.g., physical therapy) and successful response to taping that mimics surgical TTT, ${ }^{19}$ and (3) TTT with distalization and medialization. Patients were excluded if they had (1) a history of patellar dislocation, (2) TTT performed in the context of a revision procedure, (3) presence of systematic musculoskeletal disorders such as rheumatoid arthritis, (4) presence of severe rotational deformities of the lower limb requiring derotation procedures, or (5) cognitive impairment or neurodegenerative diseases.

Patellar maltracking with patella alta was defined as anterior knee pain that has an increased TT-TG distance, an increased CDI, and a good response on McConnell taping ${ }^{19}$ in which the patella is simulated to track more medially and distally. Surgery was recommended only for patients who met these criteria and in addition had insufficient response to previous conservative treatment including physical therapy for at least 6 months.

All participating patients provided their informed consent for inclusion. All study procedures were in accordance with the Declaration of Helsinki ${ }^{20}$ and were approved by the institutional ethics and review board (M010-048).

\section{Surgical Technique and Postoperative Rehabilitation}

A single surgeon (M.H.J.S.), experienced in performing TTT procedures, or a registrar under direct supervision of this surgeon, performed all procedures. Distomedialization of the tibial tubercle was performed in all knees. Preoperative planning was done for all patients through radiographic imaging and magnetic resonance imaging (MRI). There were no standard definitions for the amount of distomedialization as this differed per patient. The goal was to restore the anatomic normal values as reported in the literature. The required distal transfer was based on correction of patella alta $(\mathrm{CDI}<1.3)$ and the required medial transfer was based on correction of TT-TG distance (TT-TG $<15$ $\mathrm{mm}$ ). An individualized surgical plan was made based on these 2 measurements and the patient's specific anatomical variances. In case of extensive patella alta, more distalization was applied. Likewise, in case of substantially increased TT-TG distance, the tibial tubercle was transferred more medially.

A longitudinal incision was made over the tibial tubercle and the peritendineum was cleaved and opened on both sides. A distal block of bone on the tibial tubercle was marked and prepared with a $4.5-\mathrm{mm}$ screw hole. Oblique cuts were made with an oscillating saw on the medial and lateral side of the fragment while the proximal and distal ends were cut straight (Fig 1A). Based on the preoperative measurements, the new position of the tibial tubercle was determined. To verify the new position, the patella was pulled caudally, while attention was given for the moment when the patella would no longer move any further anteriorly. Simultaneously, tracking of the patella through the trochlea was checked for stability by manual assessment of resistance to lateral manipulation. Once stable patellofemoral tracking was observed, the definitive transposition site was marked and a second bony block was cut out, such that this aligned with the measurements and tracking (Fig 1B). The 2 bony blocks were then switched so that the tibial tubercle, with the patellar tendon attached, was transferred and countersunk into the cutout of the second block of bone and vice versa (Fig 1C). After manually assessing and approving the new patellar tracking, final fixation was achieved with a partially treated cancellous screw through the tibial tuberosity (Fig 1D). This screw penetrated the posterior tibial cortex to ensure maximal pull-out resistance. Only the tibial tubercle was fixated with a screw. Figure 2A shows a preoperative radiograph of a left knee with patella alta. Figure $2 \mathrm{~B}$ shows a radiograph of the same knee 3 months postoperatively following distomedial TTT.

Postoperatively, a custom-made knee immobilizing brace, made of synthetic cast, was applied for 6 weeks, and partial weight-bearing was allowed up to $50 \%$ of 
Fig 1. Tibial tubercle distomedialization in a right knee with patellar maltracking and patella alta. (A) Osteotomy of the tibia tubercle (arrow) with oblique saw cuts. (B) Marked position of the preoperatively planned distomedialization (arrow) for the tibia tubercle with the patellar tendon attached (asterisk), and osteotomy of the second bone block. (C) The second bone block is countersunk in the cutout of the tibia tubercle (arrow). (D) The tibial tubercle is transferred medially and distally and fixated with a partially treated cancellous screw (arrow). The second bone block is mechanically fixated in the cutout of the tibial tubercle (asterisk).

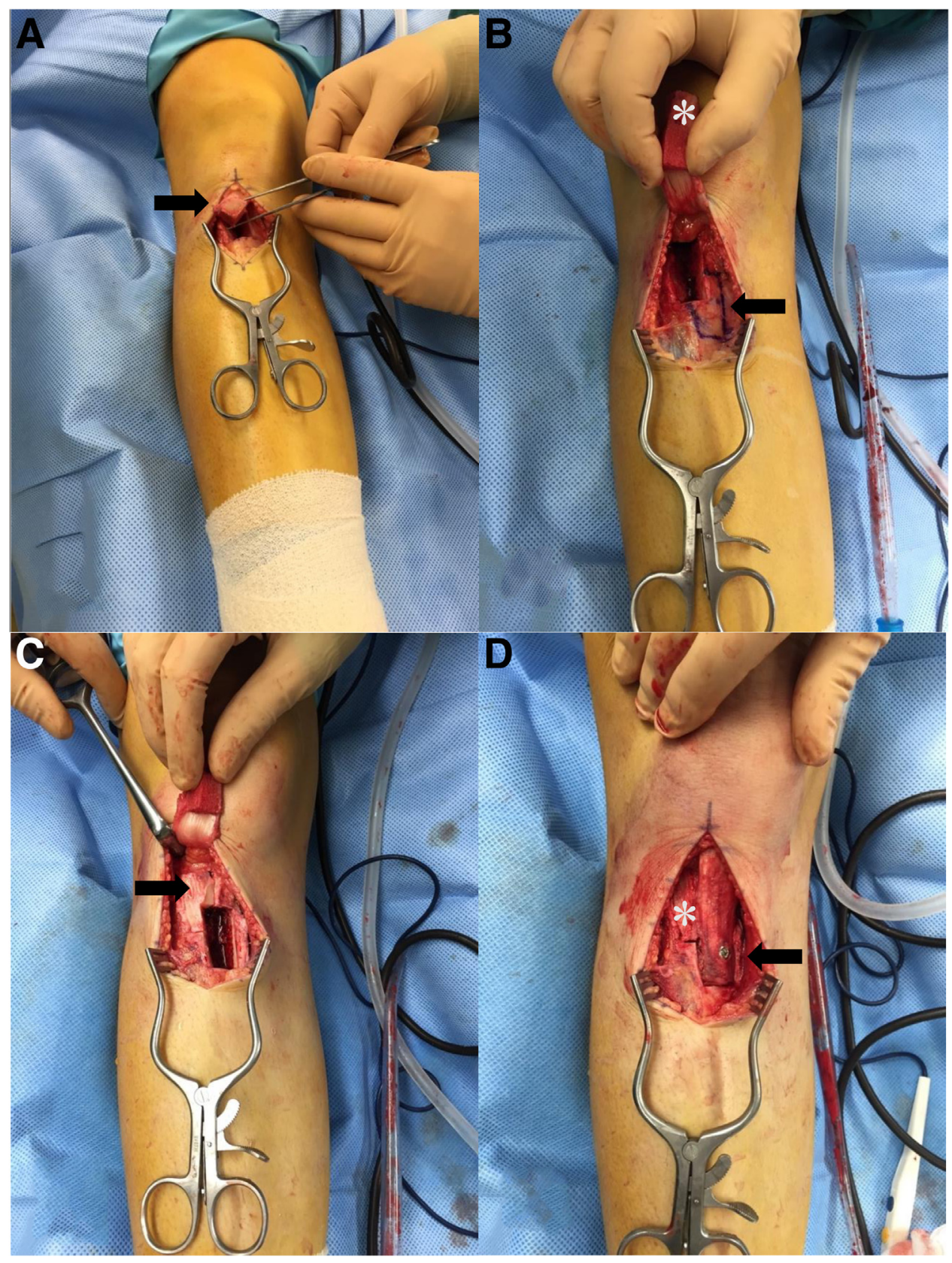

body weight with crutches. After 6 weeks, the cast was removed, and range of motion and active quadriceps exercises were commenced under the guidance of a physical therapist, provided that patellar position and signs of progressive healing were radiographically confirmed. Full weight-bearing was allowed when muscular control was regained. If consolidation of the osteotomy was confirmed on radiographs, and clinical examination did not reveal other impediments, return to low-impact sports was allowed after 12 weeks. Highimpact and contact sports were allowed after 5 to 6 months postoperatively, depending on clinical recovery.

\section{Clinical and Patient-Reported Outcomes}

Before their surgery, all patients underwent preoperative clinical evaluations and completed baseline patient-reported outcome measures (PROMs). Radiologic assessment was done for all patients through a standard routine including anteroposterior and lateral views of the knee, used to measure patellar height. In addition, magnetic resonance imaging (MRI) of the affected knee was performed to evaluate any patellofemoral chondral defects, to check for soft-tissue injuries, and for measurement of TT-TG distance. TT-TG distance was measured according to standard methods on MRI, with the knowledge that TT-TG on MRI 


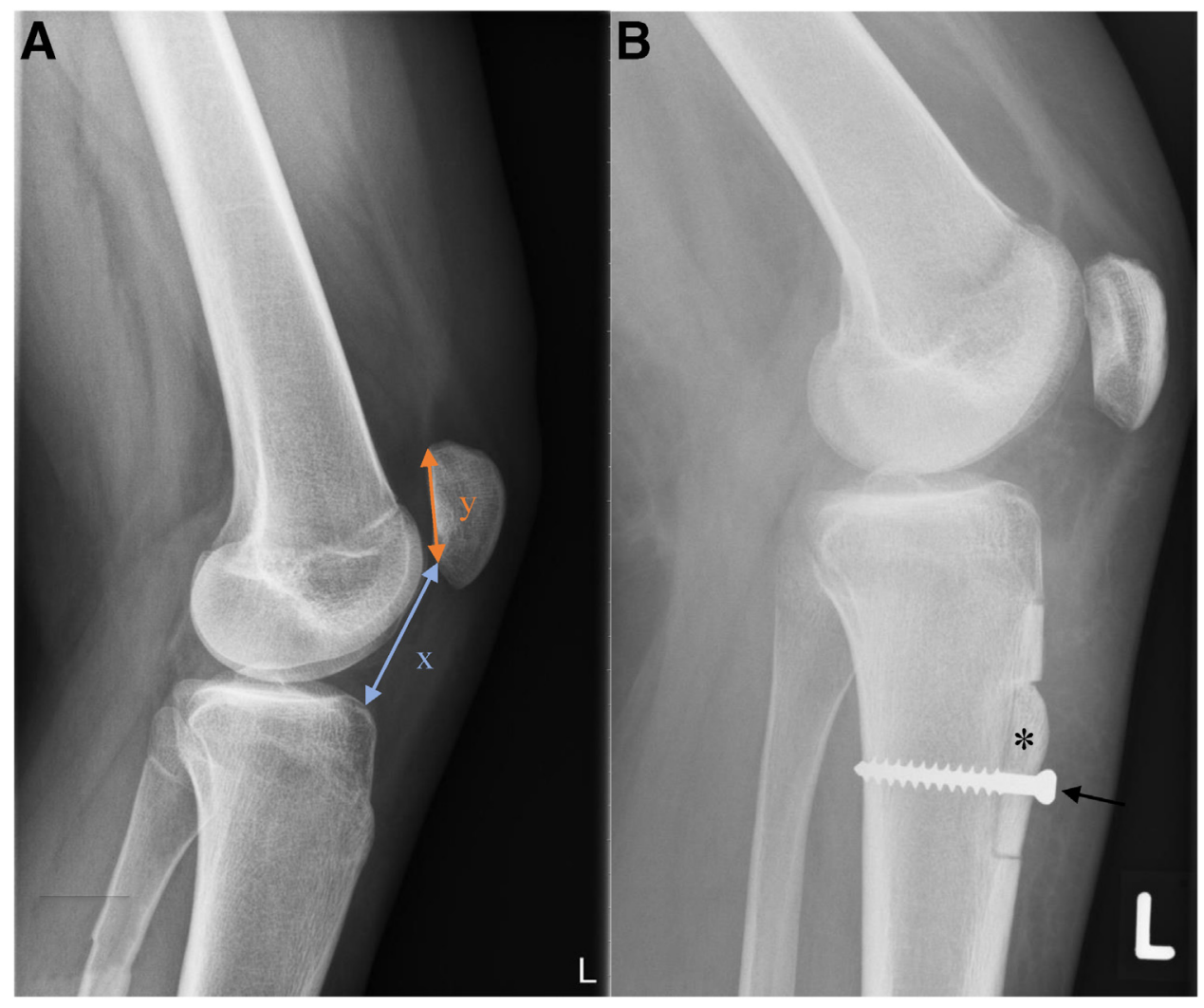

Fig 2. (A) Preoperative radiograph of a left knee with patella alta. Patella alta was defined as CDI $>1.3$. This index is measured as the ratio between the distance from the tibial articular surface to the lowest point of articular cartilage on the patella $(x)$, to the length of the articular surface of the patella $(\mathrm{y})$. CDI $=\mathrm{x} / \mathrm{y}$. (B) Radiograph of the same knee 3 months postoperatively following distomedial transfer of the tibial tubercle (asterisk) with partially treated cancellous screw fixation (arrow). (CDI, Caton-Deschamps Index.)

underestimates TT-TG on computed tomography with mean $3.5 \mathrm{~mm}^{21}$ Chondral damage was classified through MRI using the Modified Outerbridge grading system. ${ }^{22}$ PROMs included the Kujala score, ${ }^{23}$ Knee injury and Osteoarthritis Outcome Score (KOOS), ${ }^{24}$ and visual analog scale (VAS) for pain. ${ }^{25}$ Follow-up measurements were performed at 3, 6, 12, and 24 months postoperatively. To determine clinical relevance and improvement, frequencies of patients with overall change scores (i.e., difference between baseline and final follow-up) exceeding minimal clinically important differences (MCIDs) for patellofemoral pain were calculated. MCIDs in Kujala score of 12 points, ${ }^{26}$ KOOS of 10 points, ${ }^{24}$ and VAS of 20 points $^{27}$ were used. Complications were assessed during the study period and frequencies of removal of hardware ( $\mathrm{ROH})$ were calculated.

\section{Statistical Analysis}

Standard descriptive statistics were used to describe patient characteristics, preoperative clinical outcomes, and PROMs scores; logistic regression explored loss to follow-up. To study improvement over time, we performed generalized estimating equations (GEE) analyses with exchangeable correlation structures. GEE models were chosen because they correct for missing values and corrections are made for the dependency of observations within one individual. ${ }^{28}$ Only patients who were followed-up for a minimum of 12 months were included in the analyses. Outcomes were Kujala score, KOOS, and VAS, hence, linear models were used. We entered time as sole factor in each model to explore recovery over specified time intervals. Results of the GEE analyses were presented as changes over the specified time interval. All analyses were performed using SPSS, version 25.0 (IBM SPSS Statistics, Armonk, $\mathrm{NY}$ ), and the level of significance was $P<.05$ (2-tailed).

\section{Results}

\section{Study Population}

A total of 40 patients with patellar maltracking and patella alta without instability met the inclusion criteria. Finally, 8 patients were lost to follow-up; thus, outcomes of 32 patients were analyzed for the purpose of this study ( $80 \%$ follow-up rate). Mean follow-up was $22 \pm 4.4$ months. Loss to follow-up was not selective to age, sex, body mass index, duration of symptoms, or chondral damage $\left(\chi^{2}=2.14\right.$, df $=6, P=.91$, Nagelkerke $\mathrm{R}^{2}=0.07$ ). Baseline characteristics of follow-up patients are presented in Table 1 . The tibial tubercle was transferred medially over a median distance of 5.0 $\mathrm{mm}$ (interquartile range [IQR] 5.0-7.0) and distally over a median distance of $15.0 \mathrm{~mm}$ (IQR 15.0-18.5) (Table 1). No cartilage procedures were performed and no large patellar lesions were noted in this group that 
Table 1. Patient Characteristics for Follow-Up Patients

\begin{tabular}{|c|c|c|}
\hline \multirow[b]{2}{*}{ Variable } & \multicolumn{2}{|c|}{ Patients $(\mathrm{n}=32)$} \\
\hline & $\mathrm{n}$ & Median (IQR) or $\mathrm{n}(\%)$ \\
\hline$\overline{\text { Age, } y}$ & 32 & $21.0(19.0-25.9)$ \\
\hline BMI & 32 & $23.2(21.2-24.5)$ \\
\hline Right knee & 32 & $17(53 \%)$ \\
\hline Duration of symptoms, $y$ & 31 & $4.0(2.2-6.0)$ \\
\hline Physical therapy, mo & 28 & $6.0(3.0-13.5)$ \\
\hline Preoperative CDI & 32 & $1.3(1.3-1.5)$ \\
\hline Postoperative CDI & 32 & $1.0(0.9-1.1)$ \\
\hline \multicolumn{3}{|l|}{ Transfer distance, $\mathrm{mm}$} \\
\hline Medial & 30 & $5.0(5.0-6.3)$ \\
\hline Distal & 30 & $15.0(15.0-18.5)$ \\
\hline
\end{tabular}

NOTE. Patient characteristics are reported as median with corresponding IQR or in number and frequencies $(\%)$.

BMI, body mass index; CDI, Caton-Deschamps index; IQR, interquartile range; MRI, magnetic resonance imaging; TT-TG, tibial tubercle-trochlear groove distance.

required such interventions. Postoperatively, median CDI was 1.0 (IQR 0.9-1.1).

\section{Improvement Over Time}

Mean Kujala score increased from $55 \pm 12$ preoperatively to $79 \pm 16(P<.001)$ at final follow-up. No further significant improvement was observed after 6 months follow-up (Table 2 and Fig 3A). Mean KOOS increased from $48 \pm 14$ preoperatively to $79 \pm 15(P<.001)$ at final follow-up. After 6 months follow-up, no further significant improvement was observed (Fig 3B). Mean pre- and postoperative final follow-up VAS score were 64 \pm 17 and $25 \pm 21(P<.001)$, respectively. No further significant improvement in VAS was observed after 3 months follow-up (Fig 3C). MCIDs for all PROMs were exceeded by mean overall improvement scores in these PROMs. A total of 27 patients $(84 \%)$ had a clinically relevant improvement of Kujala score, 29 patients $(91 \%)$ of KOOS, and 25 patients (78\%) of VAS.

\section{Complications}

Complications occurred in 1 patient (3\%), a 16-yearold female, who suffered from anterior knee pain postoperatively for which a re-osteotomy with further medialization of the tibial tubercle and lateral retinacular release was performed 1 year after the original procedure. After this procedure, the patient continued to have anterior knee pain that required $\mathrm{ROH} 1$ year later. The patient was satisfied with the final result. A total of $72 \%$ of patients required $\mathrm{ROH}$ because of complaints of the screw. No other complications such as instability, failure of materials, joint stiffness, infection, or nonunion occurred.

\section{Discussion}

The main findings of this study were that distomedial TTT led to good functional recovery and effective pain reduction up to 24 months' follow-up in patients with patellar maltracking and patella alta without instability. Mean overall improvement in PROMs were larger than the aforementioned MCIDs, indicating clinically relevant improvement. Patient-reported outcomes improved significantly in the first 3 months when regarding the VAS, and 6 months when regarding Kujala and KOOS score, and these results remained stable over time. Furthermore, few surgical

Table 2. Mean and Change Scores and GEE Results Specified per Time Interval

\begin{tabular}{|c|c|c|c|c|}
\hline \multirow[b]{2}{*}{ Outcome } & \multicolumn{4}{|c|}{ Patients $(\mathrm{n}=32)$} \\
\hline & $\mathrm{n}$ & $\begin{array}{l}\text { Score } \\
\pm \text { SD }\end{array}$ & Change \pm SD & $P$ Value \\
\hline \multicolumn{5}{|l|}{ Kujala } \\
\hline Baseline & 32 & $55 \pm 12$ & & \\
\hline$\Delta \mathrm{T} 0-\mathrm{T} 3 \mathrm{~m}$ & 20 & $67 \pm 19$ & $-11.4 \pm 19$ & $<.001^{*}$ \\
\hline$\Delta \mathrm{T} 3-\mathrm{T} 6 \mathrm{~m}$ & 18 & $76 \pm 16$ & $-9.3 \pm 19$ & $.04^{*}$ \\
\hline$\Delta \mathrm{T} 6-\mathrm{T} 12 \mathrm{~m}$ & 16 & $79 \pm 14$ & $-3.2 \pm 12$ & .27 \\
\hline$\Delta \mathrm{T} 12-\mathrm{T} 24 \mathrm{~m}$ & 26 & $79 \pm 16$ & $0.7 \pm 17$ & .83 \\
\hline \multicolumn{5}{|l|}{ KOOS } \\
\hline Baseline & 32 & $48 \pm 14$ & & \\
\hline$\Delta \mathrm{T} 0-\mathrm{T} 3 \mathrm{~m}$ & 20 & $70 \pm 18$ & $-22.0 \pm 18$ & $<.001^{*}$ \\
\hline$\Delta \mathrm{T} 3-\mathrm{T} 6 \mathrm{~m}$ & 18 & $78 \pm 18$ & $-8.4 \pm 18$ & $.05^{*}$ \\
\hline$\Delta \mathrm{T} 6-\mathrm{T} 12 \mathrm{~m}$ & 16 & $78 \pm 15$ & $-0.1 \pm 13$ & .99 \\
\hline$\Delta \mathrm{T} 12-\mathrm{T} 24 \mathrm{~m}$ & 26 & $79 \pm 15$ & $-1.1 \pm 16$ & .73 \\
\hline \multicolumn{5}{|l|}{ VAS } \\
\hline Baseline & 30 & $64 \pm 17$ & & \\
\hline$\Delta \mathrm{T} 0-\mathrm{T} 3 \mathrm{~m}$ & 20 & $24 \pm 17$ & $40.3 \pm 22$ & $<.001^{*}$ \\
\hline$\Delta \mathrm{T} 3-\mathrm{T} 6 \mathrm{~m}$ & 18 & $22 \pm 18$ & $1.7 \pm 20$ & .72 \\
\hline$\Delta \mathrm{T} 6-\mathrm{T} 12 \mathrm{~m}$ & 17 & $24 \pm 10$ & $-2.0 \pm 22$ & .72 \\
\hline$\Delta \mathrm{T} 12-\mathrm{T} 24 \mathrm{~m}$ & 26 & $25 \pm 21$ & $-1.1 \pm 30$ & .85 \\
\hline \multicolumn{5}{|c|}{$\begin{array}{l}\text { Mean scores and change scores of outcomes and GEE results are } \\
\text { presented per time interval with corresponding SD. } \\
\text { GEE, generalized estimating equation; KOOS, Knee injury and } \\
\text { Osteoarthritis Outcome Score; m, months; SD, standard deviation; } \\
\text { VAS, visual analog scale for pain. } \\
\text { *Statistically significant. }\end{array}$} \\
\hline
\end{tabular}



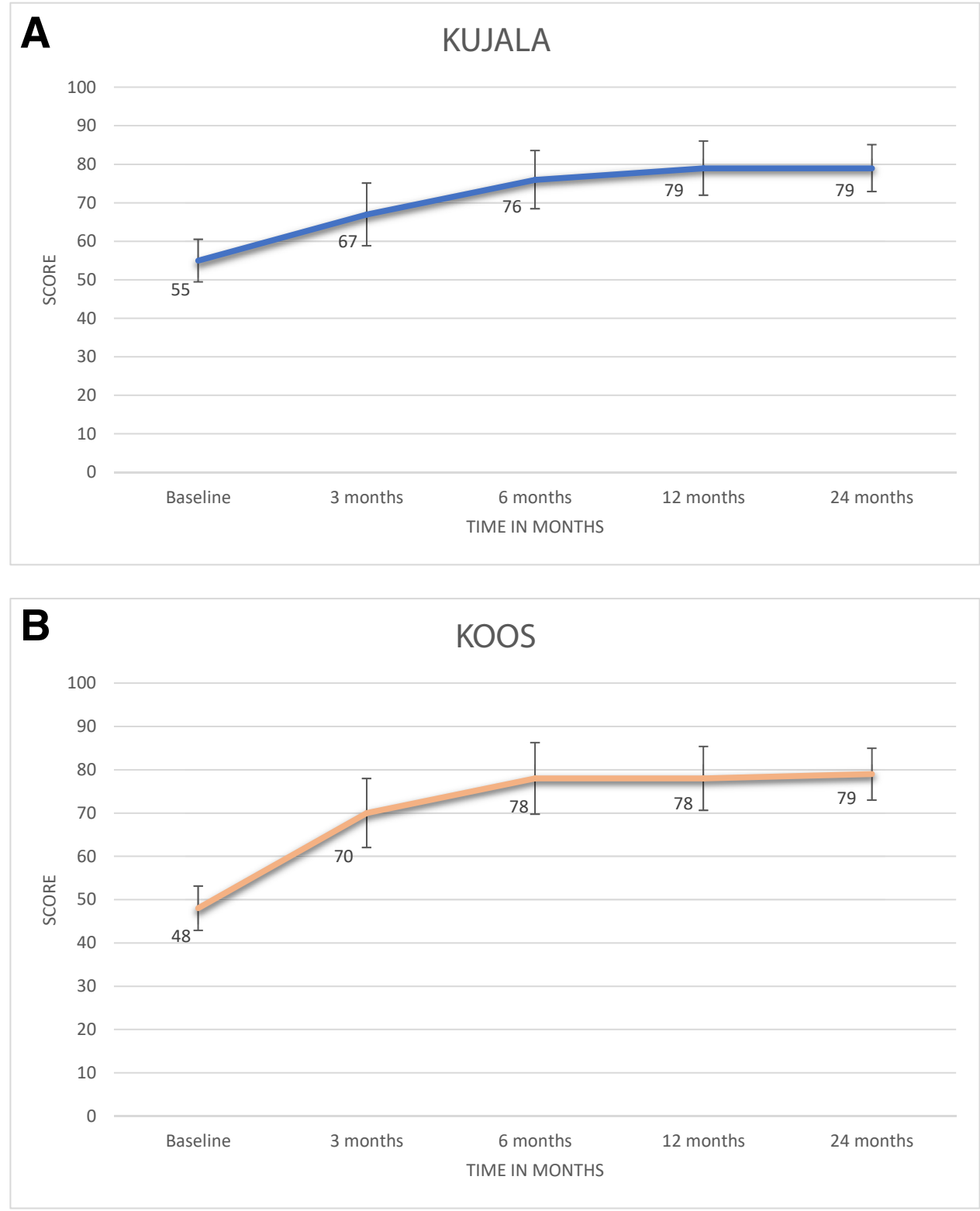

Fig 3. Overall mean results over time in: (A) Kujala. (B) KOOS. C (VAS pain score). (KOOS, Knee injury and Osteoarthritis Outcome Score; VAS, visual analog scale for pain.) complications occurred $(3 \%)$, suggesting that TTT is a safe procedure. However, the $\mathrm{ROH}$ rate was high $(72 \%)$.

This study is one of the few to prospectively assess outcomes following distomedial TTT in patients with patella alta. In a previous prospective study, Al-Sayyad et al. ${ }^{4}$ obtained corresponding results following distal transfer with significant functional improvement, few complications (7\%) and high patient satisfaction $(88 \%)$ in 25 patients with patellofemoral pain and patella alta. Although the 2-year improvement in Kujala score was similar, Al-Sayyad et al. ${ }^{4}$ differed from our study by only distalizing the insertion of the patella tendon, without performing medialization. Median TT-TG distance in the current study was $15.5 \mathrm{~mm}$, indicating significant lateral patellar tracking was present in the study population. Because lateral patellar tracking was not reported by Al-Sayyad et al. ${ }^{4}$ the study populations could not be compared. In a retrospective, comparative cohort study, Enea et al. ${ }^{17}$ performed distomedialization of the tibial tubercle in 24 patients with patella alta and either objective patellar instability or potential patellar instability. They reported improvement of functional scores and pain score comparable with the present study, without significant differences between the 2 patient groups. However, like our study, group sizes were small in the study by Enea et al., ${ }^{17}$ and to have sufficient power to detect a pre- to postoperative difference exceeding the MCID in Kujala score, larger sample sizes are needed. Tigchelaar et al. ${ }^{29}$ 
and Koëter et al. ${ }^{30}$ prospectively assessed 60 knees with patellar maltracking or instability (31 of them with patella alta) of the same study cohort, following medialization (and distomedialization in case of patella alta) at 2 years and 10 years follow-up. They found similar improvement in functional outcomes and pain scores, and demonstrated that initial improvements were maintained at 10 years follow-up, with no significant differences between the maltracking and instability group.

All aforementioned studies reported an average improvement exceeding the MCID regarding the Kujala score or VAS and these improvements only differed marginally between the studies., ${ }^{4,17,29}$ Therefore, it seems that the specific surgical technique does not influence the outcome of a TTT. However, none of the previous studies, ${ }^{4,29}$ reported change over time in outcome scores. Based on the results of the present study, patients can be informed to expect quick postoperative improvement in pain up to 3 months, and functional improvement up until 6 months following distomedial TTT. Furthermore, a total of $84 \%$ to $91 \%$ of patients had clinically relevant improvement in functional scores, and $78 \%$ of patients had clinically relevant pain relief. However, despite functional improvement and pain reduction were obtained in the vast majority of patients, a percentage as great as $22 \%$ of patients continued to have pain, and $9 \%$ to $16 \%$ of patients did not have clinically relevant functional improvement. It is therefore important to perform careful patient selection based on clinical findings and underlying anatomic variances, and to initiate nonoperative treatment first before considering distomedial TTT. In contrast, when initiating conservative therapy, it should be kept in mind that this is to avoid operative management and not to delay surgical intervention. Therefore, when considering duration and extent of conservative therapy, anatomical variances underlying patellar maltracking should be appreciated.

To address the variety of anatomical conditions associated with patellar maltracking, numerous techniques for TTT have been introduced, including medialization, distalization, anteromedialization, and distomedialization. ${ }^{5}$ Anteromedialization is primarily reserved for patients with patellar maltracking or instability with presence of partial- or full-thickness patellar lesions and aims to offload patellofemoral cartilage. ${ }^{31,32}$ In patients with patellar maltracking and patella alta, the goal of surgery is to restore patellar height and lateral tracking to normal indices to improve patellofemoral engagement. ${ }^{17,33}$ This should increase patellofemoral contact surface and reduce peak stress and pain. ${ }^{34}$ Hence, in this specific population, we feel distomedial transfer is an appropriate technique to restore patellofemoral congruence. Moreover, it should be noted that because of the slope of the proximal tibia, distal transfer also causes a certain amount of medialization of the tibial tubercle, which can be adjusted as required according to the patient's TT-TG distance. ${ }^{35}$ Distomedial transfer has initially been described by Hauser for treatment of patellar instability. This treatment was later criticized because of high complication rates and development of patellofemoral osteoarthritis. ${ }^{36,37}$ However, the procedure performed in those studies $^{36-38}$ was substantially different from the modern procedure as an overcorrection of distomedial transfer occurred with a medialization of $25 \mathrm{~mm}$ and distalization of 25 to $38 \mathrm{~mm} .{ }^{13}$ In contrast, we performed only subtle transfer of the tibial tubercle and reported a medialization of $5 \mathrm{~mm}$ and a distalization of $15 \mathrm{~mm}$ in our study. Overcorrection was avoided through preoperative estimation of transfer distance, based on radiologic measurements, and final intraoperative control of the new patellar tracking before the definitive transposition site was determined.

Currently, concerns related to long-term complications such as degenerative joint disease following distomedial TTT remain. Up to 24 months postoperatively, only a few complications $(3 \%)$ occurred following distomedial TTT in this study. However, follow-up may have been too short for osteoarthritic changes to manifest, and no postoperative MRIs were performed to evaluate this. Tigchelaar et al. ${ }^{29}$ reported long-term incidence of postoperative patellofemoral degeneration that was similar to the natural history of conservatively treated patellar instability. One may argue that with corresponding subtle medial transfer distance, a comparable incidence of postoperative osteoarthritic change in patients of the present study could be expected.

Furthermore, in a biomechanical study, Luyckx et al. ${ }^{34}$ demonstrated that patella alta is associated with reduced patellofemoral contact surface but higher patellofemoral contact pressure compared with the normal height patella. These findings were in accordance with similar biomechanical ${ }^{8}$ and radiologic studies $^{6,9,39}$ that showed an association between patella alta and reduced patellofemoral contact surface. Moreover, patella alta was associated with an elevated prevalence of patellofemoral osteoarthritis resulting from reduced patellofemoral loadbearing surface and subsequent increased peak stress. ${ }^{9,39}$ It can therefore be stated that correction of patella alta to a "normal" height, as is pursued with distomedial TTT, allows for an increased loadbearing surface and subsequently for decreased peak stress as result of improved pressure distribution. Distomedial TTT may consequently be efficacious in prevention, or reduction of progression of patellofemoral cartilage degeneration in patients with patella alta. Nevertheless, long-term studies are needed to confirm the incidence of patellofemoral osteoarthritis following distomedial TTT. 
Despite good clinical results following the procedure, the $\mathrm{ROH}$ rate in this study was high $(72 \%)$. Previous studies reported $\mathrm{ROH}$ rates ranging from $10 \%$ to $96 \%$ following different techniques for TTT. ${ }^{16}$ The relatively high rate in this study can be attributed to the large compression screw that was used in this series. In the future, smaller AO screws can be placed to avoid this. Furthermore, during standard follow-up, patients were asked actively for any complaints of the screw, which might have led to a greater $\mathrm{ROH}$ rate. We consider the $\mathrm{ROH}$ rate an important disadvantage of the procedure. However, patients who have suffered from long-term anterior knee pain due to patellar maltracking and patella alta may experience few disadvantages of $\mathrm{ROH}$ relatively to the clinical benefit of TTT. Nevertheless, adaption of technique and materials to reduce the $\mathrm{ROH}$ rate should be explored.

\section{Limitations}

This study was subject to several limitations. First, PROMs were not completed by all patients on every follow-up, and lost to follow-up was present. Intermittent missing data and loss to follow-up limited the ability to assess change in outcomes over time on larger scale and may have given rise to selection bias. However, we aimed to correct this limitation by performing GEE analyses and demonstrated that loss to follow-up was not selective for baseline patient characteristics. Second, the 24-month follow-up period allowed only for short-term effects of the procedure to manifest, and on the base of this study, long-term results could only be hypothesized. Therefore, no hard statements regarding incidence of postoperative arthritic changes could be made. Finally, because of the character of the study, no comparison group was involved. Hence, outcomes of the procedure could not be compared with results of conservatively treated patients.

\section{Conclusions}

In this case series study, distomedial TTT led to clinically relevant functional improvement and pain reduction in patients with patellar maltracking and patella alta without instability. However, the ROH rate was high $(72 \%)$.

\section{References}

1. Thomeé R, Augustsson J, Karlsson J. Patellofemoral pain syndrome: A review of current issues. Sports Med 1999;28: 245-262.

2. Colvin AC, West RV. Patellar instability. J Bone Jt Surg Am 2008;90:2751-2762.

3. Magnussen RA, De Simone V, Lustig S, Neyret P, Flanigan DC. Treatment of patella alta in patients with episodic patellar dislocation: A systematic review. Knee Surg Sports Traumatol Arthrosc 2014;22:2545-2550.
4. Al-Sayyad MJ, Cameron JC. Functional outcome after tibial tubercle transfer for the painful patella alta. Clin Orthop Relat Res 2002;396:152-162.

5. Hall MJ, Mandalia VI. Tibial tubercle osteotomy for patello-femoral joint disorders. Knee Surg Sports Traumatol Arthrosc 2016;24:855-861.

6. Ward SR, Terk MR, Powers CM. Patella alta: Association with patellofemoral alignment and changes in contact area during weight-bearing. J Bone Joint Surg Am 2007;89: 1749-1755.

7. Charles MD, Haloman S, Chen L, Ward SR, Fithian D, Afra R. Magnetic resonance imaging-based topographical differences between control and recurrent patellofemoral instability patients. Am J Sports Med 2013;41:374-384.

8. Ward SR, Powers CM. The influence of patella alta on patellofemoral joint stress during normal and fast walking. Clin Biomech (Bristol, Avon) 2004;19:1040-1047.

9. Stefanik JJ, Zhu Y, Zumwalt AC, et al. Association between patella alta and the prevalence and worsening of structural features of patellofemoral joint osteoarthritis: The multicenter osteoarthritis study. Arthritis Care Res (Hoboken) 2010;62:1258-1265.

10. Jibri Z, Jamieson P, Rakhra KS, Sampaio ML, Dervin G. Patellar maltracking: an update on the diagnosis and treatment strategies. Insights Imaging 2019;10:65.

11. Iliadis AD, Jaiswal PK, Khan W, Johnstone D. The operative management of patella malalignment. Open Orthop $J$ 2012;6:327-339.

12. Sherman SL, Erickson BJ, Cvetanovich GL, et al. Tibial tuberosity osteotomy: Indications, techniques, and outcomes. Am J Sports Med 2014;42:2006-2017.

13. Hauser ED. Total tendon transplant for slipping patella: A new operation for recurrent dislocation of the patella. 1938. Clin Orthop Relat Res 2006:452:7-16.

14. Pritsch T, Haim A, Arbel R, Snir N, Shasha N, Dekel S. Tailored tibial tubercle transfer for patellofemoral malalignment: Analysis of clinical outcomes. Knee Surg Sports Traumatol Arthrosc 2007;15:994-1002.

15. Longo UG, Rizzello G, Ciuffreda M, et al. Elmslie-Trillat, Maquet, Fulkerson, Roux Goldthwait, and other distal realignment procedures for the management of patellar dislocation: Systematic review and quantitative synthesis of the literature. Arthroscopy 2016;32:929-943.

16. Bayoumi T, Benner JL, Stavenuiter MHJ, van der List JP. Tibial tubercle transfer leads to clinically relevant improvement in patients with patellar maltracking without instability: A systematic review and meta-analysis [published online June 27, 2020]. Knee Surg Sports Traumatol Arthrosc. https://doi.org/10.1007/s00167-0002006114-00161.

17. Enea D, Canè PP, Fravisini M, Gigante A, Dei Giudici L. Distalization and medialization of tibial tuberosity for the treatment of potential patellar instability with patella alta. Joints 2018;6:80-84.

18. Phillips CL, Silver DA, Schranz PJ, Mandalia V. The measurement of patellar height: A review of the methods of imaging. J Bone Joint Surg Br 2010;92:1045-1053.

19. Leibbrandt DC, Louw QA. The use of McConnell taping to correct abnormal biomechanics and muscle activation patterns in subjects with anterior knee pain: A systematic review. J Phys Ther Sci 2015;27:2395-2404. 
20. Association WM. World Medical Association Declaration of Helsinki: Ethical principles for medical research involving human subjects. JAMA 2013;310:2191-2194.

21. Hinckel BB, Gobbi RG, Filho EN, et al. Are the osseous and tendinous-cartilaginous tibial tuberosity-trochlear groove distances the same on CT and MRI? Skeletal Radiol 2015:44:1085-1093.

22. Paunipagar B, Rasalkar D. Imaging of articular cartilage. Indian J Radiol Imaging 2014;24:237-248.

23. Kievit AJ, Breugem SJM, Sierevelt IN, et al. Dutch translation of the Kujala Anterior Knee Pain Scale and validation in patients after knee arthroplasty. Knee Surg Sports Traumatol Arthrosc 2013;21:2647-2653.

24. Roos EM, Lohmander LS. The Knee injury and Osteoarthritis Outcome Score (KOOS): From joint injury to osteoarthritis. Health Qual Life Outcomes 2003;1:64-64.

25. Hawker GA, Mian S, Kendzerska T, French M. Measures of adult pain: Visual Analog Scale for Pain (VAS Pain), Numeric Rating Scale for Pain (NRS Pain), McGill Pain Questionnaire (MPQ), Short-Form McGill Pain Questionnaire (SF-MPQ), Chronic Pain Grade Scale (CPGS), Short Form-36 Bodily Pain Scale (SF-36 BPS), and Measure of Intermittent and Constant Osteoarthritis Pain (ICOAP). Arthritis Care Res (Hoboken) 2011;63:S240-S252 (suppl 11).

26. Agarwalla A, Liu JN, Wu HH, Kalbian IL, Garcia GH, Shubin Stein BE. Return to work following tibial tubercle osteotomy for patellofemoral osteoarthritis and pain [published online April 22, 2020]. Cartilage. doi: 1947603520916544.

27. Crossley KM, Bennell KL, Cowan SM, Green S. Analysis of outcome measures for persons with patellofemoral pain: Which are reliable and valid? Arch Phys Med Rehabil 2004;85:815-822.

28. Twisk JW. Longitudinal data analysis. A comparison between generalized estimating equations and random coefficient analysis. Eur J Epidemiol 2004;19:769-776.

29. Tigchelaar S, Van Essen P, Bénard M, Koëter S, Wymenga A. A self-centring osteotomy of the tibial tubercle for patellar maltracking or instability: Results with ten-years' follow-up. Bone Joint J 2015;97-B: 329-336.
30. Koëter S, Diks MJ, Anderson PG, Wymenga AB. A modified tibial tubercle osteotomy for patellar maltracking: Results at two years. J Bone Joint Surg $\mathrm{Br}$ 2007;89:180-185.

31. Fulkerson JP, Becker GJ, Meaney JA, Miranda M, Folcik MA. Anteromedial tibial tubercle transfer without bone graft. Am J Sports Med 1990;18:490-496. discussion 496-497.

32. Pidoriano AJ, Weinstein RN, Buuck DA, Fulkerson JP. Correlation of patellar articular lesions with results from anteromedial tibial tubercle transfer. Am J Sports Med $1997 ; 25: 533-537$.

33. Diks MJ, Wymenga AB, Anderson PG. Patients with lateral tracking patella have better pain relief following CT-guided tuberosity transfer than patients with unstable patella. Knee Surg Sports Traumatol Arthrosc 2003;11: 384-388.

34. Luyckx T, Didden K, Vandenneucker H, Labey L, Innocenti B, Bellemans J. Is there a biomechanical explanation for anterior knee pain in patients with patella alta? Influence of patellar height on patellofemoral contact force, contact area and contact pressure. J Bone Joint Surg 2009;91:344-350.

35. Batailler C, Lustig S, Servien E. Tibial tubercle osteotomies: Techniques and Distalization. In: Dejour D, Zaffagnini S, Arendt EA, Sillanpää P, Dirisamer F, eds. Patellofemoral pain, instability, and arthritis: clinical presentation, imaging, and treatment. Berlin, Heidelberg: Springer Berlin Heidelberg, 2020;277-282.

36. Chrisman OD, Snook GA, Wilson TC. A long-term prospective study of the Hauser and Roux-Goldthwait procedures for recurrent patellar dislocation. Clin Orthop Relat Res 1979:27-30.

37. Barbari S, Raugstad TS, Lichtenberg N, Refvem D. The Hauser operation for patellar dislocation. 3-32-year results in 63 knees. Acta Orthop Scand 1990;61:32-35.

38. Crosby EB, Insall J. Recurrent dislocation of the patella. Relation of treatment to osteoarthritis. J Bone J Surg Am 1976;58:9-13.

39. Haj-Mirzaian A, Guermazi A, Pishgar F, et al. Association of patella alta with worsening of patellofemoral osteoarthritis-related structural damage: Data from the Osteoarthritis Initiative. Osteoarthr Cartil 2019;27:278-285. 